\title{
EFFECT OF LEAKAGE ON VELOCITY AND PRESSURE DISTRIBL IN PIPELINES NETWORK
}

Shaimaa Rashed, Osama Saleh, Magdy Mowafy

\begin{abstract}
Controlling water losses through leakage in water supply systems is an important issue for perfect water management. There are generous studies have been investigated, trying to get close to the ideal design of water pipelines network system. Also perfect applicable of the system in the field, as well as having a good management for the system for several years. The existing paper may response about partial requests in that field. The purpose of this paper is to investigate the effect of leakage that may occur in pipeline systems on the pressure and velocity. Various factors, influencing water losses have been identified and investigated. The dynamic behaviors that have been investigated in the paper were; the effect of the local energy loss including leakages on the pressure, velocity or discharge in pipelines network, and the effect of water pressure in pipelines on the leakage rate
\end{abstract}

